\title{
Initial Survey to Fungal Deterioration of Archaeological Linen Textiles in Sohag National Museum
}

\author{
Mahmoud Abo Elmaaref ${ }^{a} *$, Mohamed Marouf ${ }^{a}$, Wael S. Mohamed ${ }^{b}$, Walaa \\ A. Abdel Wahab \\ a. Conservation Department, Faculty of Archeology, Sohag University, Egypt. \\ b. Polymers Department, National Research Centre, Dokki, Giza, Egypt. \\ c. Department of Chemistry of Natural and Microbial Products, National Research Centre, Dokki, Giza, Egypt.
}

\section{HIGHLIGHTS}

- The dominant fungi isolated and identified from the display and storage atmosphere in the Sohag National Museum were Aspergillus flavus, Trichoderma sp., and Penicillium duclauxii.

- The identified fungi affected the physical, chemical and mechanical properties of archaeological linen textiles.

- The most dangerous fungal strains affect all properties studied was Aspergillus flavus.

\section{ARTICLE INFO}

\section{Article History:}

Received: 29 September 2020

Revised: 22 October 2020

Accepted:31 October 2020

Available online: 21 December 2020

Keywords:

Microbial surveys, CIE Lab, FTIR, tensile strength, color change.

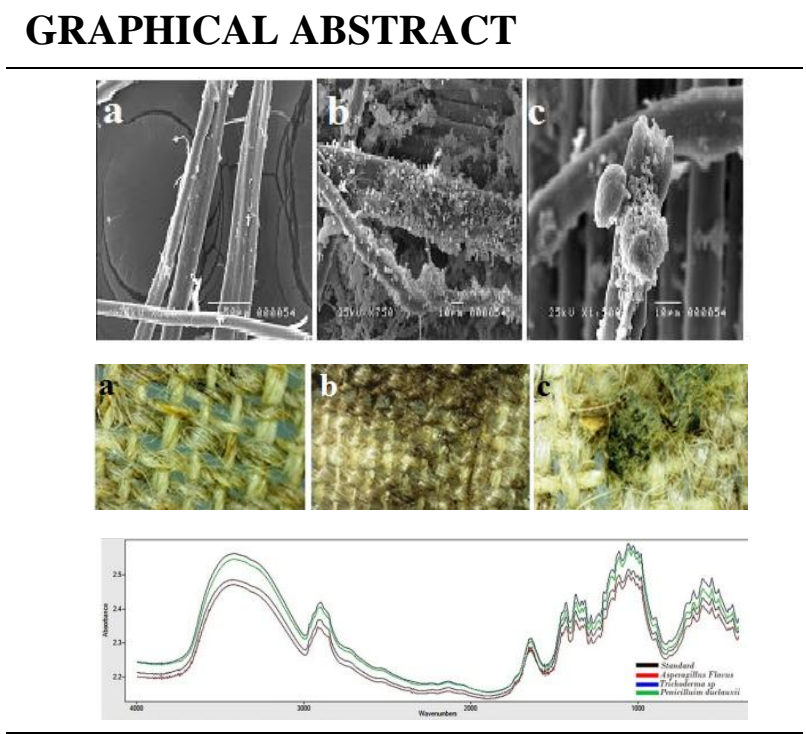

\begin{abstract}
Microorganisms are one of the most dangerous damage factors that threaten archaeological cellulosic textiles such as cotton and linen. This research presents a detailed study of the effect of fungi that have been isolated from the display and storage environment of Sohag National Museum on the mechanical, physical and chemical properties of archaeological linen textiles. The study used the potato Dextrose Agar, PDA. Nutritional medium in conducting microbial surveys of the museum display and storage environment; the result of the survey was the presence of three fungal strains, Aspergillus flavus, Trichoderma sp. and Penicillium duclauxii, which dominated the display and storage atmosphere in the museum.
\end{abstract}

* Corresponding author: dr_aboelmaaref@yahoo.com 
The study evaluated the effect of these fungal strains on the linen textiles. Measurement of mechanical properties (tensile strength and elongation), change of color using the CIE Lab system and the use of Fourier transform infrared spectroscopy (FTIR) were used for the evaluation process. The results of this study found that the most dangerous fungal strain that affect both mechanical, physical properties and chemical composition was strain of Aspergillus flavus.

\section{Introduction}

This study focuses on the effect of microorganisms isolated from the atmosphere of the exhibition and the museum storage of Sohag National Museum on the mechanical, physical and chemical properties of archaeological linen textiles. Organic museum collections are damaged by microorganisms as a result of multiple reasons and contributing factors, among which are the display conditions (i.e. temperature, relative humidity, and light) within museums, as well as the display methods since having a proper display system within museums preserves museum collections as well as presents them in an archaeological and scientific form which greatly benefits visitors and researchers; however, this is not the case in most museums and excavations where storage and display conditions are inappropriate. Each of the previous factors plays a significant role in the deterioration cycle of organic monuments within the museums. These factors combined cause severe damage to the collections until they reach a state of weakness and fragility. This usually leads to the destruction and loss of these collectibles [1]. Microorganisms attack textiles, which results in rapid degradation of textile fibers. Microorganisms pose a threat to textile materials at all stages of its manufacture; from obtaining raw material from farms to storage and transportation stages of the final product, where under favorable conditions for the growth of these organisms, their impact on textile fibers and materials is greater and faster [2]. The attack of microorganisms is destructive to historical textiles since such organisms remain latent for very long periods within the material of the fabric and during which microbes work to reproduce.Microbial damage can occur in collectibles that appear to be in a good condition and exist under controlled surrounding conditions; that is free of any pollutants, chemi- cal damage factors and physical pressures [3]. The prevention of microbial damage due to improper display or storage in museums, which promote fungal growth, is one of the major problems in the maintenance and treatment of archaeological textiles [4]. Microbial damage to archaeological textiles leads to color change, staining, distinct odor, and rot as a result of the secretion of these organisms as volatile compounds, in addition to the occurrence of enzymatic and mechanical degradation resulting from the activity of the growth of fungi [5]. The innate damage to textiles also works to change the properties of textiles such as a change of tensile strength and durability, in addition to the fact that some fungi contain colored materials that spot and stain textile collectibles. Moreover, these stains contain chemicals that cause damage to archaeological textiles even with the death of these fungi. Accordingly, maintaining valuable textiles requires the removal of such stains, given that the chemical changes resulting from the activity of such microorganisms lead to a strong decrease in the tensile strength of the fabrics and occurrence of a total and partial destruction of the fibrous material. Furthermore, mold from this fungal growth is also extremely harmful to those working in these museums, sometimes leading to serious diseases [4], [6]. Cellulosic fibers from cotton and linen are considered one of the most susceptible textile fibers to the attacks of microorganisms, but this does not exclude exposure of protein textiles such as wool and silk to such a risk. The mold may also grow on industrial fibers such as nylon and polyester if these fibers are soiled or due to the presence of processing materials which create a nutrient-rich environment suitable for the growth of these microorganisms [7]. Bacteria and fungi destroy textiles and fibers, but the damage from bacteria is much more harmful to fibers. Among the most significant bacte- 
rial strains which deleteriously affects textiles and their materials are the following species: Cytophaga, Micrococcus, Bacterium, Bacillus, Cellulobacillus, Pseudomonas, Sarcina, while the most damaging and harmful fungi strains to fabric materials present in the air and soil are: Aspergillus, Penicillium, Alternaria, Cladosporium, Fusarium, Trichoderma $[8,9]$. Many researchers were interested in monitoring fungal strains that dominated the archaeological linen textiles found in the Coptic Museum and the Egyptian Museum in Cairo, and the monitoring showed the most dominant strains are as follows, respectively: Aspergillus, Penicillium, Chaetomium, Alternaria, Trichoderma virida. The ability of textiles to absorb and restore their moisture content from the surrounding environment with the nature of their organic components makes them the most sensitive to the damage caused by microorganisms. There are many reasons why textiles are more sensitive to microbial growth processes, among which is that textile materials are a suitable food source for these microorganisms due to their organic nature. Moreover, the huge changes that may occur in the properties of textiles as a result of natural aging processes make them more sensitive to microbial damage [10]. Microorganisms convert carbohydrates with large insoluble molecules into soluble substances by breaking these molecules into smaller soluble units caused by Exoenzymes produced by these microorganisms and then digested by the Endoenzyme which produced within the microbial cell [11]. Natural polymers such as cellulose, starch, and protein are completely degraded by enzymes produced by microorganisms such as cellulase decomposes cellulose enzymes, amylase decomposes starch, and a protease enzyme decomposes proteins. The degradation of these polymers results in new substances that represent the vital component of microbial cell food for microorganisms, while industrial polymers are almost very stable in the environment and are not exposed to such enzymatic degradation by microorganisms [12].

\section{Materials and methods}

\subsection{Samples and chemicals}

- Raw linen produced by The Egyptian Textile Industries Company (Dintex) in Cairo.

- Potato dextrose agar (PDA) produced by (HIMEDIA REF) company used as the nutritional medium of the fungi.

\subsection{Microbial survey for the exhibition and storage environment of Sohag National Museum}

In this part, microflora of the textile exhibition and storage halls of Sohag National Museum was isolated in order to identify the most dominant fungi in the atmosphere of the museum's exhibition and storage halls. The nutritional medium of the fungus, Potato dextrose Agar (PDA) which consists of $4.0 \mathrm{~g}$ Potato Starch, 20g Dextrose, 15g Agar, processed in $1000 \mathrm{ml}$ distilled water was chosen [13]. The culture media was then sterilized in the plant disease laboratory in the Faculty of Agriculture of the University of Sohag with the Autoclave device. The culture media was poured and the antibiotic Magnabiotic 1200 mg was added upon casting to prevent the growth of bacteria on the medium used. The culture medium was then poured in Petri dishes that were transferred to the museum. A microbial survey was conducted in the display and storage halls of the museum. The microflora process was carried out by exposing 2 Petri dishes containing culture medium in the textile display halls of the museum and exposing a number of other Petri dishes in the storage halls of the National Museum of Sohag. Fig. 1 shows the microflora sampling process of the display and storage environment of Sohag National museum.

\subsection{Processing of raw linen samples, cultivating isolated fungal strains and microscopy of the fiber surface}

In this part, samples of raw linen fibers prepared and processed by the Egyptian Textile Industries Company "Dintex" in Cairo, were washed repeatedly with hot water and soap to get rid of impurities, processing materials and starch that can be found on the fibers. 
Then, the fibers were left to dry at room temperature. Upon the completion of the processing phase, linen was cut into $15 \mathrm{~cm} \times$ $3 \mathrm{~cm}$ samples in preparation for mechanical and physical tests and chemical analyses. This was followed by the cultivation stage which involved cultivating the fungal strains of fungi that have been isolated and purified from the microbial study that was carried out on the galleries and storage of the Sohag National Museum. This stage was carried out as follows:-

The cultivation medium selected was an airtight glass box that was first sterilized using ethyl alcohol, followed by heat sterilization using a Bunsen burner. After the sterilization stage, the samples were sterilized using the Autoclave to ensure that they are free of any microbial activity. The samples were then placed inside the sterile glass box and sprayed with distilled water. Three fungal strains were brought in and each fungus was transferred to the samples using an inoculation needle and then placed in the glass box. Samples were placed in a sterile Petri dish and incubated at a temperature of between 25-28 ${ }^{\circ} \mathrm{C}$ for 7-14 days [14]. The following image (Fig. 2) shows the process of inoculating raw fungal strains onto linen samples inside the glass box, which was used as an alternative to the laminar flow device which is used in the isolation, identification, purification and cultivation of fungus.

\subsection{Evaluation of the effect of fungal growth on linen textile samples by tests and analyses:-}

The process of evaluating the effect of the fungal growth of fungi isolated from the atmosphere of display and storage areas of the National Museum of Sohag on raw linen samples relied on a series of mechanical and physical tests, in addition to chemical analyses as follows:

\subsubsection{Mechanical test (Elongation and maximum force)}

The measurement of tensile strength and elongation of experimental samples was conducted at the National Institute of Standards (NIS) in Giza. The measurement process was carried out using a US Tinius Olsen device with strength of $5 \mathrm{kN}$. Test conditions were under $25^{\circ} \mathrm{C}$ and $65 \%$ relative humidity, the distance between the jaws of the device was $5 \mathrm{~cm}$, and the dimensions of the samples were $3 \times 15 \mathrm{~cm}$. The measurement was done according to international standards (ASTM D503506 (2008) standard test method for breaking force and elongation of textile fabrics (strip method).

\subsubsection{Measurement of color change with the CIELAB system}

The measurement of the values of color change of linen samples infected with fungi was measured at the National Institute of Standards in Giza using OPTIMATCH 3100. Total color change $\left(\Delta \mathrm{E}^{*}\right)$ of fungal infected linen samples was calculated according to the following equation:

$\Delta \mathrm{E}^{*}=\left\{\left(\Delta \mathrm{L}^{*}\right)^{2}+\left(\Delta \mathrm{b}^{*}\right)^{2}+\left(\Delta \mathrm{a}^{*}\right)^{2}\right\}^{1 / 2}[14,15$, 16].

\subsubsection{Fourier transform infrared spec- troscopy (FTIR)}

The analysis of samples was carried out by the infrared spectrometer to identify functional groups of the samples. Sample preparation involves grinding the sample in the form of powder, adding potassium bromide $\mathrm{KBr}$ and then performing further grinding. The powder is then compressed to make a transparent disc by placing the sample in a hollow ring half an inch in diameter and applying pressure for at least two minutes. The sample was then immediately placed in the device to avoid contamination or steam absorption [16].

\section{Results and Discussion}

\subsection{Microbial survey (Microflora) of the exhibition and storage environ- ment of Sohag National Museum-}

The Microscopic and cultural characteristics of the isolates were observed. The fungal isolates belong to the genera Aspergillus sp., Trichoderma sp., and Penicillium sp. The fungal species were identified and characterized based on their morphological characters and microscopic analysis using taxonomic guides and standard procedures [17] 

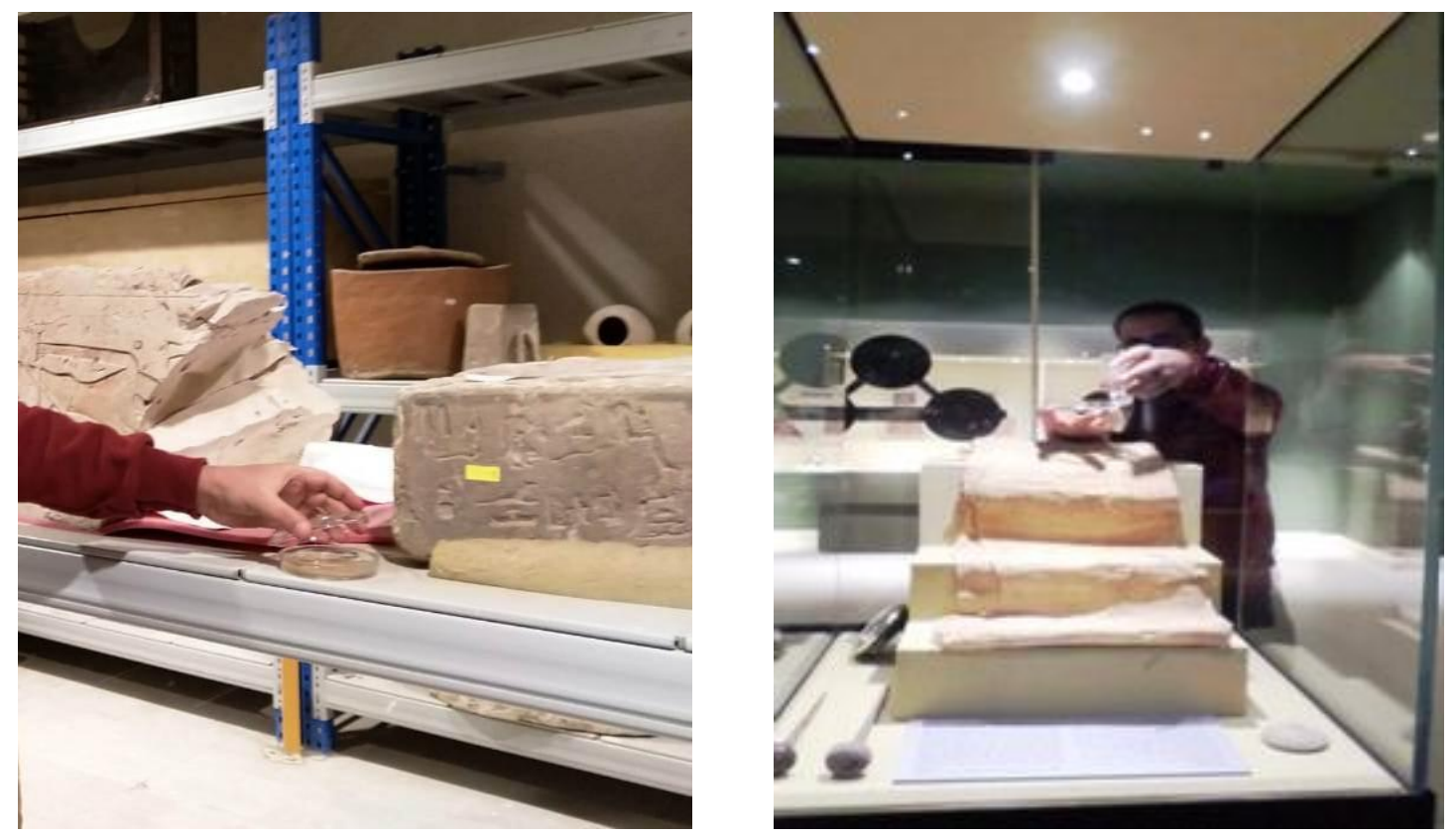

Fig. 1. Microbial surveys of the display and storage environment at the Sohag National Museum.

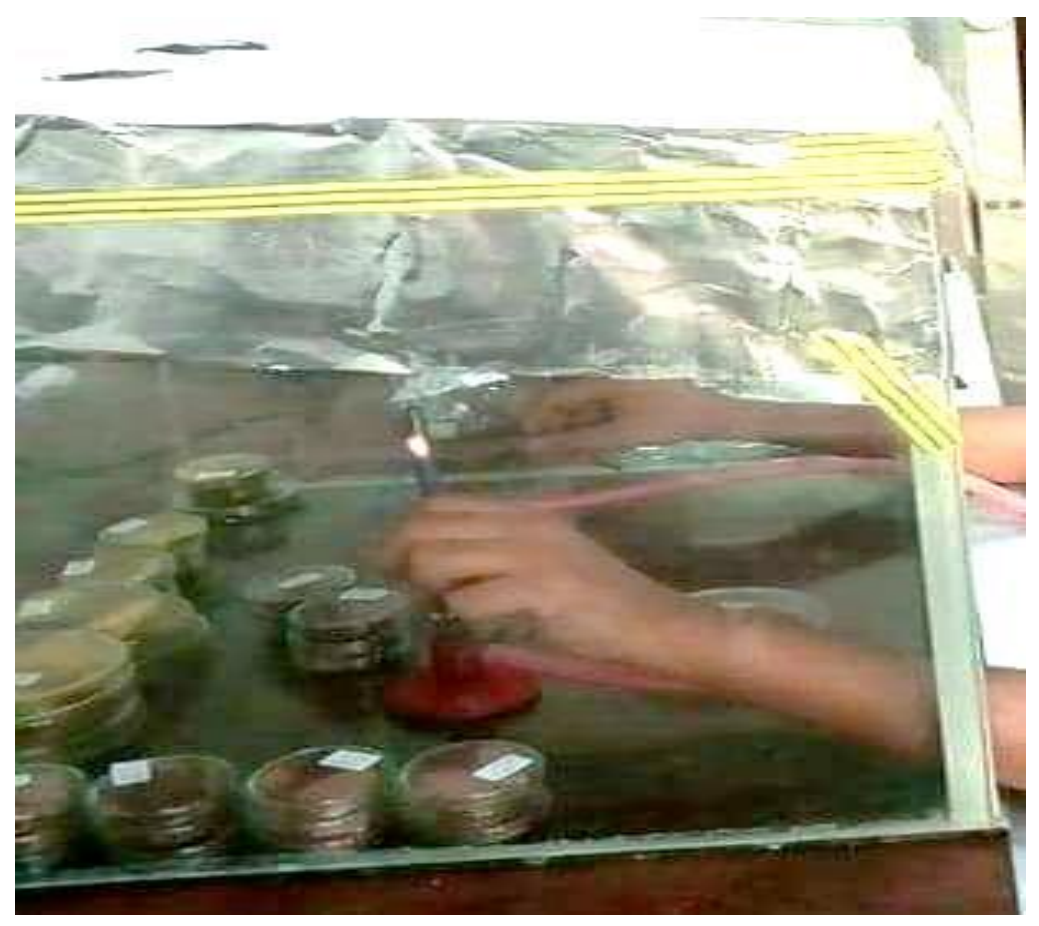

Fig. 2. The process of cultivating fungal strains on raw linen samples at the Organic Archaeology laboratory of the Faculty of Archaeology Sohag University. 
at the Microbiology Department, the National Institute of Oceanography and Fisheries, Red Sea branch, Egypt. The results of the microbial survey of fungi found in the galleries and the museum storage of the National Museum of Sohag showed the existence of three fungal strains dominated the exhibition and storage environment. These fungal strains have been represented in the exhibition halls in three types of fungi, namely 6 strains of Aspergillus flavus, one strain of Trichoderma sp. and one strain of Penicillium duclauxii. Moreover, 11 fungal strains of the museum's storage environment were represented in three types of fungi, namely 4 strains of Aspergillus flavus, 4 strains of Trichoderma $s p$. and 3 strains of Penicillium duclauxii. The dominant fungi were confined to the display and storage environment of three fungal strains, namely Aspergillus flavus, Trichoderma sp, and Penicillium duclauxii. The following images (Fig. 3) show the fungal strains that dominated the atmosphere of display and storage in the museum after being purified.

It was noted that the most dominant and present fungal strains in the display and storage environment in the museum is the fungal strain of Aspergillus flavus, where 6 fungal strains were isolated from the display environment as well as 4 fungal strains from the storage environment. It was noted that the number of fungal strains isolated from the storage environment was greater than the fungal strains isolated from the halls of the exhibition and this is may be due to the lack of proper maintenance, as well as the poor environmental conditions in the Museum store (i.e. high relative humidity and warm air) which create a climate suitable for the growth of microorganisms.

\subsection{Processing of raw linen samples, cultivating isolated fungal strains and microscopy of the fiber surface:}

The samples were examined post the cultivation of fungal strains using a stereomicroscope and a Scanning Electron Microscope. The following images show propagation and staining samples with fungal strains.
SEM microscopy showed the rapid spread and growth of fungal conidia on raw linen samples as evident in the following images:Examination of the infected samples using the stereoscopic microscope as in Fig. 4, and the SEM as in Fig. 5 show a significant distortion in the morphology of flax fibers as a result of the rapid and increasing growth of isolated fungal strains.

\subsection{The effect of fungal growth on the mechanical, physical, and chemical properties of linen fibers:-}

\subsubsection{Mechanical tests (tensile strength and elongation):}

The test of the mechanical properties of the samples (i.e. tensile strength and elongation) show the existence of a direct relationship between the strength of the fiber tension and its elongation as this is observed in the decrease in tensile rates directly followed by a decrease in the elongation of the samples compared to the standard raw linen sample. The mentioned samples of raw experimental linen infected with fungal strains isolated from the display, and storage environment recorded a decrease in the strength of the tension and elongation of the samples as shown in Fig. 6.

As a result of the growth of these fungal strains, the imbalance in the tensile strength and the elongation of linen samples, a strong decrease in the samples is observed, as shown in Fig. 6, where the fungal strain of Aspergillus flavus was the most affected in tensile and elongation testing of the samples compared to the standard sample, the sample of linen infected with Aspergillus flavus fungus $26.4005 \mathrm{~kg} / \mathrm{f}$ and elongation $1.076 \%$ which is the lowest in the strength of the tension and elongation compared to the raw sample. The standard sample strength was $54.1469 \mathrm{~kg} / \mathrm{f}$ and was $1.813 \%$. This indicates the severe effect of the fungus on the mechanical properties of cellulose fibers, specifically linen fibers. 

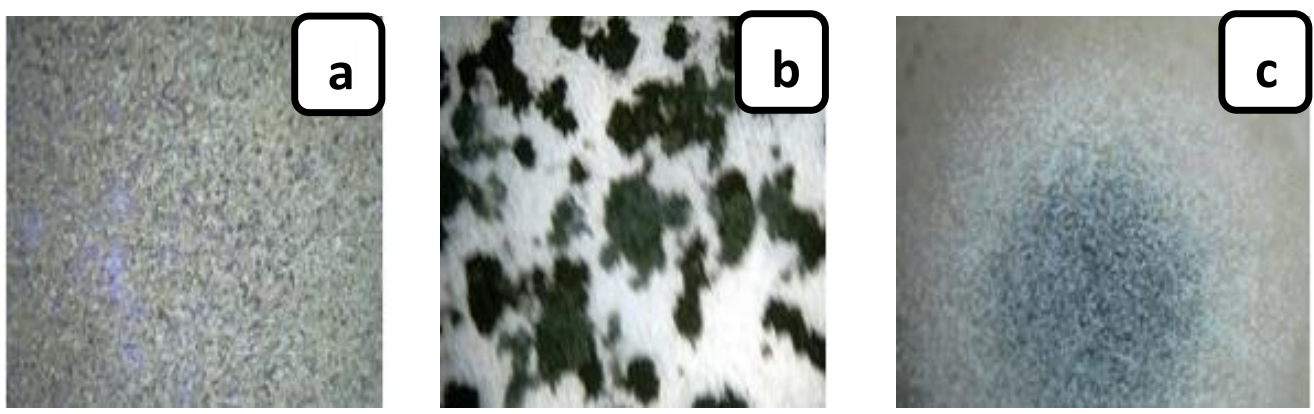

Fig. 3. The fungal strains that dominate the display and storage environment of the Sohag National Museum: (a) Aspergillus flavus, (b) Trichoderma sp (c) Penicillium duclauxii. Images taken using a USB light microscope
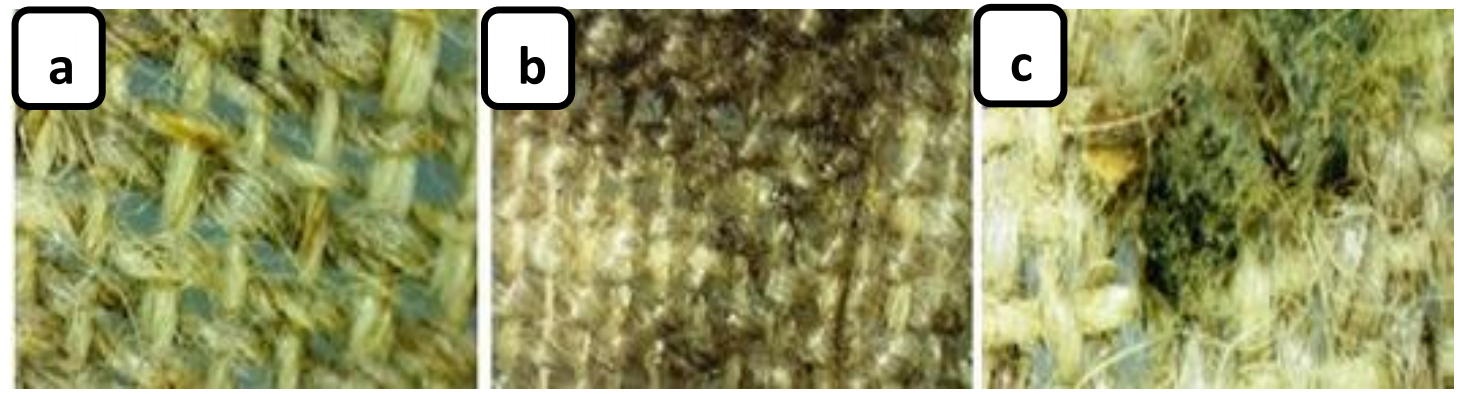

Fig. 4. Images taken using a stereo microscope showing the microbial growth on the surface of raw linen samples, where (a) represents a raw linen sample (10x), (b,c) represent a sample of linen infected with Aspergillus flavus (30x).
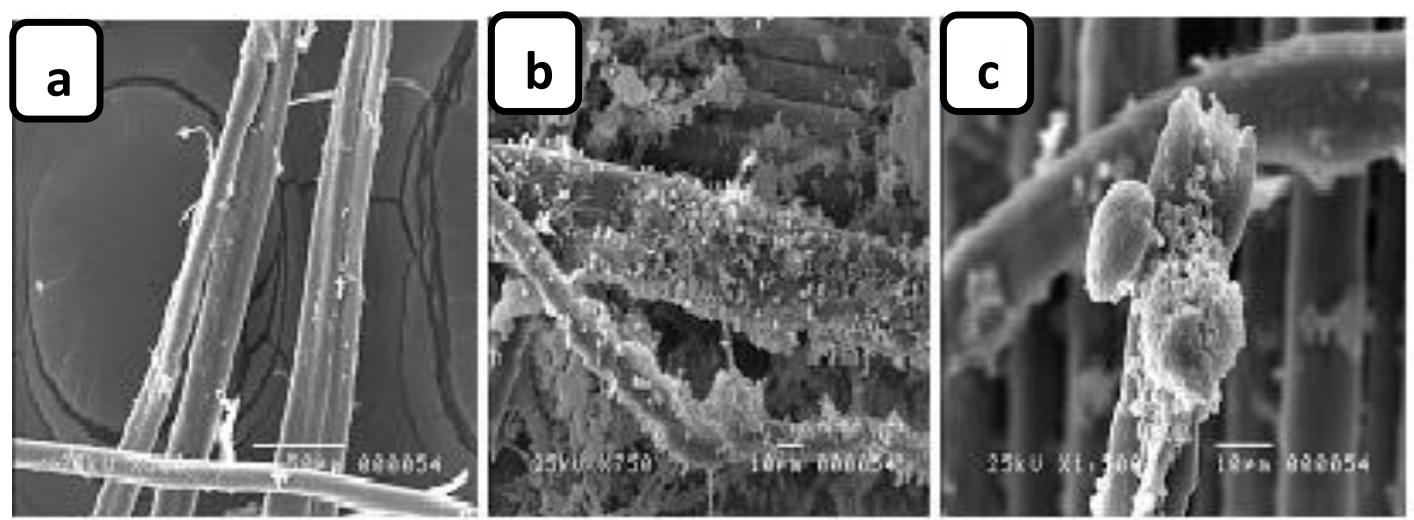

Fig.5. SEM images of the fungal conidia on raw linen samples, (a) raw linen sample shows the characteristic goffer of flax fibers $(500 x)$, (b at $1000 x$, and c at 1500x) a fungi-infected linen sample that clearly shows fungal conidia and spores. 
3.3.2. Measurement of color change with the CIELAB color system):-

The results obtained (Table 1) show changes in the values of the $\mathrm{L}^{*}, \mathrm{a}^{*}$, and $\mathrm{b}^{*}$ color coordinates of the samples as a result of fungal growth. Significant change in the value of total color change $\left(\Delta \mathrm{E}^{*}\right)$ was observed for the infected samples. It is clear from the table that the most affecting fungal strains in terms of color change are the samples infected with fungal strain of Aspergillus flavus.

Table 1. The total color difference of linen samples infected with fungi

\begin{tabular}{|c|c|c|c|c|c|}
\hline $\mathrm{n}$ & Sample & $\Delta \mathrm{L}^{*}$ & $\Delta \mathrm{a}^{*}$ & $\Delta b^{*}$ & $\Delta \mathrm{E}^{*}$ \\
\hline 1 & $\begin{array}{l}\text { Raw linen in- } \\
\text { fected with } \\
\text { Aspergillus } \\
\text { flavus }\end{array}$ & -5.35 & 0.33 & 4.36 & 6.91 \\
\hline 2 & $\begin{array}{l}\text { Raw linen in- } \\
\text { fected with } \\
\text { Trichoderma sp }\end{array}$ & -1.02 & 0.12 & 1.42 & 1.75 \\
\hline 3 & $\begin{array}{l}\text { Raw linen in- } \\
\text { fected with } \\
\text { Penicillium } \\
\text { duclauxii }\end{array}$ & -3.03 & 0.20 & 0.54 & 3.08 \\
\hline
\end{tabular}

Fungal spots on the surface of the samples also resulted in a change in the physical properties of the samples, represented by color changes. Table (1) illustrates the values of color change of raw linen samples infected with fungi Aspergillus flavus, Trichoderma, Penicillium duclauxii, where the $\Delta \mathrm{L}^{*}$ values refers to difference in the brightness axis $\mathrm{L}^{*}$ before and after fungal growth [18]. From the results of the previous table, it is clear that the $\Delta \mathrm{L}^{*}$ values for all samples indicate the darkening of the samples, and the rates of linen darkness were increased in samples with fungal spots, particularly the sample infected with Aspergillus flavus. As for the $\Delta \mathrm{a}^{*}$ values, they reflect the difference in the red-green axis, where the negative $\Delta \mathrm{a}^{*}$ value indicates the shift of the sample towards a more green color. On the other hand, the pos- itive $\Delta \mathrm{a}^{*}$ value indicates the shift of the sample towards a more red color [19]. Table 1 shows that the sample of linen infected with Aspergillus flavus has recorded the highest value of change in the $\Delta \mathrm{a}^{*}$ color coordinate (i.e. $\Delta \mathrm{a}^{*}=0.33$ ), where the value of $\Delta \mathrm{a}^{*}$ for the sample was 0.29 . The $\Delta \mathrm{b}^{*}$ value reflects the difference in the yellow-blue axis, as the positive $\Delta b^{*}$ value indicates the shift of the sample towards a more yellow color, while the negative $\Delta b^{*}$ value indicates the shift of the sample towards a more blue color. According to the results listed in Table 1, the yellowing of the samples infected with Aspergillus flavus fungus increased, with a $\Delta \mathrm{b}^{*}$ value of 4.36 , followed by the Trichoderma infected linen sample with a $\Delta \mathrm{b}^{*}$ value of 1.42. The table also shows an increase in the total color change $\Delta \mathrm{E}^{*}$ for linen samples infected with fungi, in particular the Aspergillus flavus sample with a $\Delta \mathrm{E}^{*}$ value of 6.91, followed by other samples infected with fungi.

\subsubsection{Fourier transform infrared spectroscopy (FTIR)}

The results obtained (Fig. 7) showed the change in the infrared spectrum of the Aspergillus flavus, Trichoderma sp., and Penicillium duclauxii infected linen sample, where the figure show the appearance of oxidation and hydrolysis products of cellulose fibers expressed in the spectral band 1900$1500 \mathrm{~cm}^{-1}$.

The FTIR spectrum of the raw linen sample infected with Aspergillus flavus shows a decrease in the spectral band at $433.62 \mathrm{~cm}^{-1}$ and an increase in the spectral band at 1095.42 $\mathrm{cm}^{-1}$, where the decrease and elevation in these ranges reflect a decrease in the rate of crystallization of cellulosic materials. The emergence of the bands at $520.08 \mathrm{~cm}^{-1}$ and $1381.42 \mathrm{~cm}^{-1}$ reflects the depolymerization process of the cellulose polymer, the process in which the cellulose polymer is transformed as a result of the breakup of existing 
bonds into units' monomers [20]. The increased vibration intensity at the spectral band $1900-1500 \mathrm{~cm}^{-1}$ is a distinct range of cellulose degradation products [21], as well as the appearance of the $1633.17 \mathrm{~cm}^{-1}$ band, a distinct range that expresses vibration in the water particles absorbed within cellulose [21] and the following table (Table 2) shows vibration in the spectral band $1900-1500 \mathrm{~cm}^{-1}$.

Table 2. Vibration in the spectral band between 1900-1500 $\mathrm{cm}^{-1}$ sample of raw linen infected with Aspergillus flavus fungus.

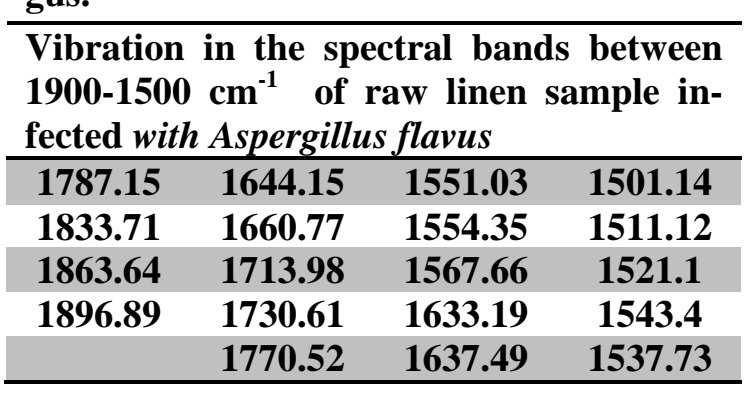

The appearance of the spectral band at $2921.18 \mathrm{~cm}^{-1}$, which is a distinct range of vibration in $\mathrm{CH}_{2}$ associated with the appearance of the carbonyl band, which expresses the degradation and damage of cellulose material [22], is also observed as the increased intensity of vibration at the spectral range $3900-3600 \mathrm{~cm}^{-1}$, which is a distinct range of $(\mathrm{OH})$ hydroxyl groups and the Table 3 shows vibration in this range.

Table 3. Vibration in the spectral band $3900-3600 \mathrm{~cm}^{-1}$ for the sample of raw linen infected with Aspergillus flavus

\begin{tabular}{|c|c|c|}
\hline \multicolumn{3}{|c|}{$\begin{array}{l}\text { Vibration in the spectral band between } \\
3900-3600 \mathrm{~cm}^{-1} \text { for the sample of raw linen } \\
\text { infected with Aspergillus flavus fungus }\end{array}$} \\
\hline 3812.45 & 3709.36 & 3626.22 \\
\hline 3849.03 & 3739.29 & 3631.52 \\
\hline 3875.67 & 3752.59 & 3656.15 \\
\hline \multirow[t]{2}{*}{3898.92} & 3755.92 & 3676.11 \\
\hline & 3802.47 & 3686.08 \\
\hline
\end{tabular}

\section{Conclusion}

The results of the study show the demanding need to pay attention to the exhibition and storage environment of the National Museum of Sohag due to its poor display and storage conditions. Such conditions include a favorable climate for the growth of microorganisms (i.e. fungi) and in particular the storage environment, as demonstrated by the results of the analyses and tests carried out on the study of the effect of microorganisms isolated from the display and storage environment on the mechanical and physical properties as well as the chemical properties of archaeological textiles. Results show that microorganisms have caused significant damage to the properties of linen represented by a decrease in the force of tensile and elongation of the samples infected with fungal, especially those infected with Aspergillus flavus, and the occurrence of many color changes in the samples as a result of increased fungal growth. The presence of such microorganisms has resulted in a significant effect on the chemical properties of linen samples.

Such damage is represented through an increase in the oxidation and degradation of cellulose as evident at the spectral range of $1900-1500 \mathrm{~cm}^{-1}$ in the resultant FTIR spectra. The presence of these fungi has also resulted in a decrease in the rate of crystallization of fiber forming cellulose.

\section{References}

1. H. E .Ahmed, S. S. Darwish, Effect of museum conditions on Historical dyed silk fabric with madder dye, Journal of Polymers and the Environment, Vol. 20, No. 2, 2012, pp. 596-606.

2. G. B. Michalski, Microbial degradation of woven fabrics and protection against biodegradation, IntechOpen, 2012, P 268. 


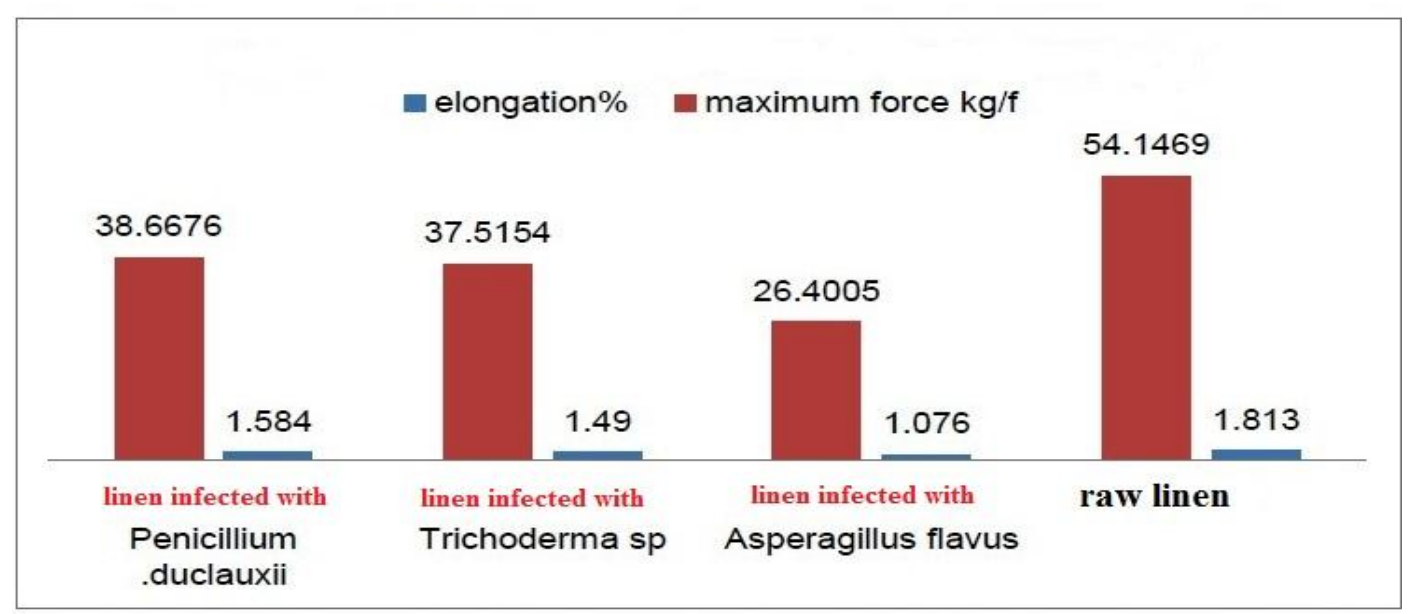

Fig. 6. Tensile strength and elongation of experimental linen samples infected with fungal strains isolated from the exhibition and storage environment of the Sohag National Museum.
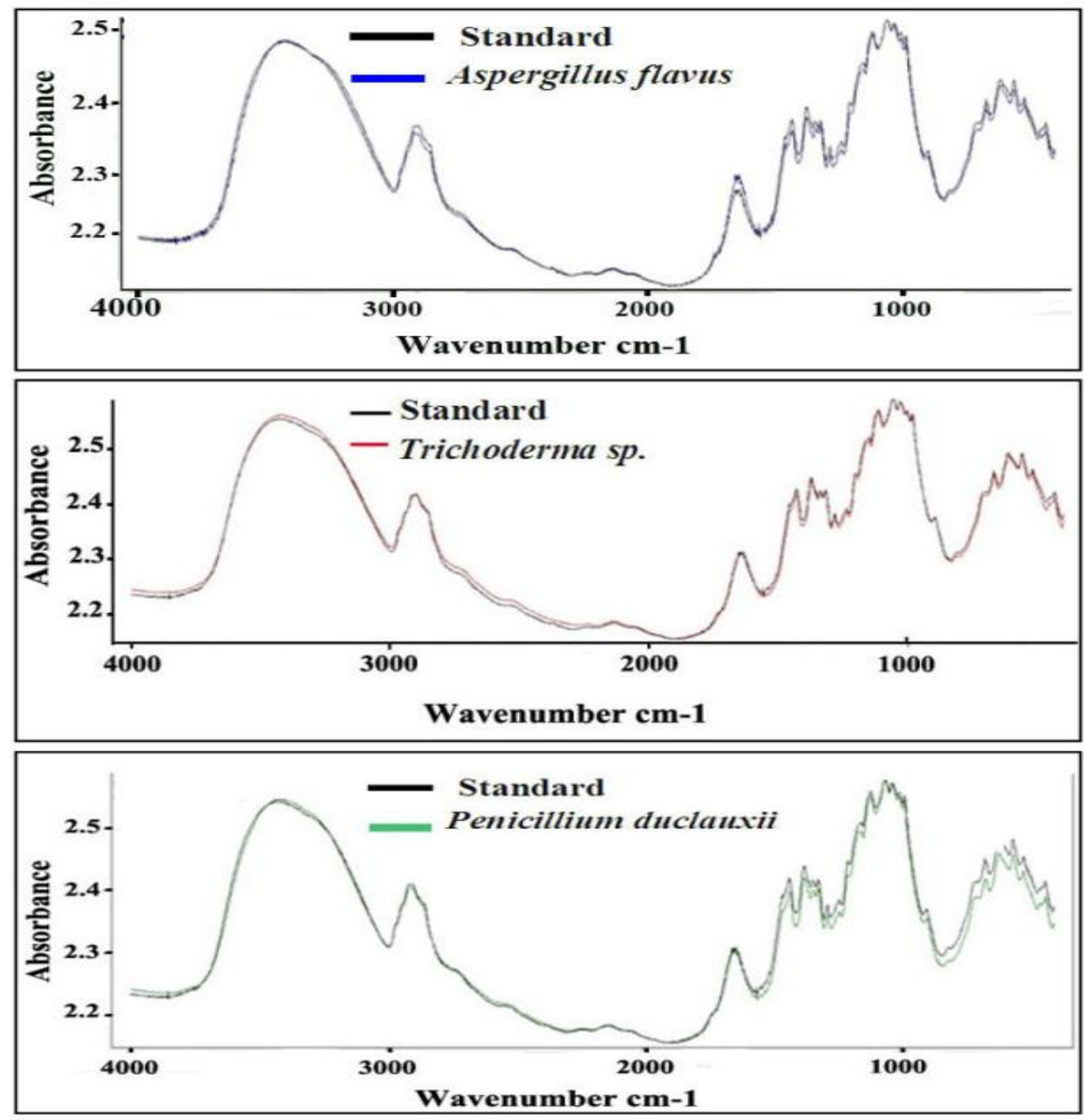

Fig.7. FTIR spectra of the raw linen samples infected with fungi Aspergillus flavus, Trichoderma, and Penicillium duclauxii 
https://pdfs.semanticscholar.org/680c/6c 859c202072205ca510a7ae6ae02d9098b $\underline{0 . p d f}$.

3. R. Mitchell, C.J. Mcnamara, Cultural heritage microbiology: Fundamental studies in conservation science, American Society For Microbiology, USA, 2010.

4. O. Abdel Kareem, Fungal deterioration of historical textiles and approaches for their control in Egypt, e-Preservation Science, Vol. 7, 2010, pp. 40-47

5. K. Kavkler, C. N. Gunde, P. Zalar, A. Demsar. Fungal Contamination Of Textile Objects Preserved In Slovene Museums And Religious Institutions, International Biodeterioration \& Biodegradation, Vol. 97, 2015. pp. 51-59.

6. J. S. Kotowa, Biodeterioration of textiles, International Biodeterioration \& Biodegradation, Vol. 53, No. 3, 2004, pp.165-170.

7. Canadian Conservation Institute CCI, Mould growth on textiles, Notes 13/15. https://www.canada.ca/en/conservationinstitute/services/conservationpreservation-publications/canadianconservation-institute-notes/mouldgrowth-textiles.html

8. E. Pekhtasheva, A. Neverov, S. Kubica,.G. Zaikov, Biodegradation and biodeterioration of some natural polymers, Chemistry \& Chemical Technology, Vol. 6, No. 3, 2012, pp. 263-280.

9. G. Abdel-Maksoud, H. Emam, N.M. Ragab, From traditional to laser cleaning techniques of parchment manuscripts: A review, Advanced Research in Conservation Science, Vol. 1, No. 1, 2020, pp. 52- 76.

10. O. Abdel Kareem, Evaluating the combined efficacy of polymers with fungicide for protection of museum textile against fungal deterioration in Egypt , polish Journal of Microbiology, Vol. 59, No. 4, 2010, pp. 271-280.

11. H. J. Flint, K. P. Scott, S. H. Duncan, P. Louis, E. Forano, Microbial degradation of complex carbohydrates in the gut, Gut Microbes, Vol. 3, No. 4, 2012, pp. 289-306.

12. K. Sakai, T. Yamauchi, F . Nakasu, T. Ohe, Biodegradation of cellulose acetate by Neisseria sicca, Bioscience, Biotechnology, and Biochemistry, Vol. 60, No. 10, 1996, pp. 1617-1622.

13. Potato dextrose agar. https://en.wikipedia.org/wiki/Potato_de xtrose_agar $\quad$ (signed $14 / 1 / 2020 \mathrm{~h}$ $10.00 \mathrm{pm})$.

14. M. Abo elmaaref, Cleaning of archaeological cellulosic textile with Enzymes and surfactant detergents "A comparative experimental and applied study, M.A thesis, Conservation Department, Faculty of Archaeology, Sohag University,2017. P.162.

15. S.F. Ibrahim, D.M. Essa, E.M. Osman, Statistical method for determining the levelness parameters of different colored polymeric fabrics, International Journal of Chemistry, Vol. 3, No. 3; 2011, pp. 11-20.

16. P.B. Pathare, U. L. Opara, F. A. AlSaid, Colour measurement and analysis in fresh and processed foods: A review, Food Bioprocess Technol, Vol. 6, 2013,pp. 36-60.

17. I. Kopecká, E, Svobodová, Methodology for infrared spectroscopy analysis of sandwich multilayer samples of historical materials, Heritage Science, Vol. 2, 2014, pp. 1-8.

18. K.H. Domsch., W. Gams, T. H. Anderson. "Compendium of soil fungi. Vol. 1, Academic Press (London) Ltd., London. 1980. Pp. 1011-1018.

19. J. Kolar, A. Stolfa, `c.M .Strli, M. Pompe, B. Pihlar,. M Budnar, c. J .Sim̌cǐ, 
B. Reissland, Historical iron gall ink containing documents Properties affecting their condition, Analytica Chimica Acta,Vol.555, No. 1, 2006, pp. 167-174.

20. L .Csefalvayová , B. Havlínová , M. Čeppan, Z .Jakubíková , The Influence of Iron Gall Ink on Paper Ageing, Restaurator, Vol. 28, No. 2, 2007, pp. 129139.

21. K. Kavkler, A. Demsar, Application of FTIR and raman spectroscopy to Qualitative Analysis of Structural changes in Cellulosic Fibres, Tekstilec, Vol. 55, No. 1, 2012, pp. 19-44.

22. V. Hospodarova, E. Singovszka, N. Stevulova, Characterization of cellulosic fibers by FTIR spectroscopy for their further implementation to building materials, American Journal of Analytical Chemistry, Vol. 9, 2018, pp. 303-310. 